\title{
Random Allocation in Controlled Clinical Trials: A Review
}

Bolaji Emmanuel Egbewale

Department of Community Medicine, Ladoke Akintola University of Technology, Ogbomoso, Nigeria

Received, February 16, 2014; Revised, May 23, 2014; Accepted, May 30, 2014; Published, June 2, 2014.

\begin{abstract}
PURPOSE: An allocation strategy that allows for chance placement of participants to study groups is crucial to the experimental nature of randomised controlled trials. Following decades of the discovery of randomisation considerable erroneous opinion and misrepresentations of its concept both in principle and practice still exists. In some circles, opinions are also divided on the strength and weaknesses of each of the random allocation strategies. This review provides an update on various random allocation techniques so as to correct existing misconceptions on this all important procedure. METHODS: This is a review of literatures published in the Pubmed database on concepts of common allocation techniques used in controlled clinical trials. RESULTS: Allocation methods that use; case record number, date of birth, date of presentation, haphazard or alternating assignment are non-random allocation techniques and should not be confused as random methods. Four main random allocation techniques were identified. Minimisation procedure though not fully a random technique, however, proffers solution to the limitations of stratification at balancing for multiple prognostic factors, as the procedure makes treatment groups similar in several important features even in small sample trials. CONCLUSIONS: Even though generation of allocation sequence by simple randomisation procedure is easily facilitated, a major drawback of the technique is that treatment groups can by chance end up being dissimilar both in size and composition of prognostic factors. More complex allocation techniques that yield more comparable treatment groups also have certain drawbacks. However, it is important that whichever allocation technique is employed, unpredictability of random assignment should not be compromised.
\end{abstract}

This article is open to POST-PUBLICATION REVIEW. Registered readers (see "For Readers") may comment by clicking on ABSTRACT on the issue's contents page.

\section{INTRODUCTION}

Randomisation is a major principle and the hallmark of randomised controlled trials (RCTs). It is a procedure that allows for chance allocation of trial participants to the treatment groups. The procedure in principle ensures that treatment groups are balanced in terms of baseline characteristics and thus provides the basis for posttreatment crude between-group comparison of treatment effect. It is widely believed that randomisation offers a good technique of controlling bias. Ever since its discovery by Fisher in 1920 and subsequent introduction to medical research by Hill in the 1940s, there have been many controversies among medical researchers regarding the principles and practices of randomisation in the context of controlled experiments [1].

To date, there is still some misconception regarding randomisation techniques and this may adversely affect the design of clinical trials [2]. It has been argued [1] that although randomisation is supposed to be fundamental to the success of any controlled clinical trial, it remains perhaps the least understood aspect of such studies. For example, researchers often confuse non-random techniques - such as haphazard allocation, alternating assignment and using date of birth - with true randomisation techniques. This review thus seeks to elucidate concepts and theories of randomisation and common strategies for allocating patients or participants to treatment groups in a clinical trial setting.

\section{METHODS}

The review was carried out in the Pubmed database. A series of steps were followed to determine the final search terms. Firstly, to conduct a concise search on this subject matter, keywords on the topic were identified. For the purpose of this search, the key words that were considered are: Random, allocation, controlled clinical trial.

Also, in the interest of an exhaustive search in the specified database, synonyms of the key words

Corresponding Author: Dr Egbewale B.E; Department of Community Medicine, Ladoke Akintola University, Ogbomoso, Nigeria; Email: begbewale@live.com 
were identified. The MeSH database was used for this purpose; the database was accessed on the Pubmed home page. The MeSH search for the first key word 'Random' shows that 'Random allocation' is a MeSH term and so therefore there are indexed citations in the Pubmed that contains the phrase. It is only good to build this into the final search terms that shall be conducted in the Pubmed. Thus, the phrase 'Random allocation' was selected as the first search term and the MeSH database was further used to build the final search in the Pubmed. Since 'allocation' exists together as a MeSH term with Random, it was decided to skip searching for the synonyms of the word and continued search was made for synonyms of the next key phrase; 'controlled clinical trial'. This phrase also exists as a MeSH term. So summarily, since the key words exist together as MeSH terms, it was decided to use the combination of those key words that appeared as MeSH terms in the final Pubmed search.

On the other hand, the use of 'wild card,' with the word random (random*) performed this search much better than the method already described. All the MeSH terms that contained the word random with indexed citation in the Pubmed were enlisted, and thus, building the search terms for the final search in the Pubmed becomes easier. There were 132 of such MeSH terms that are synonyms or have to do with random. They appeared as single word or phrase. Most are not actually relevant to this review anyway. Following the identification of relevant MeSH terms, each of the other MeSH terms was combined to build the final search terms that was used for the final search in the Pubmed database. After following appropriate links, the first search strategy produced the following combination of MeSH terms: "Random Allocation"[Mesh] AND "Randomised Controlled Trial "[Publication Type] and when search was made in the Pubmed database a very large number ( 17484) of indexed citations that included the concept being searched were indicated. The search strategy was however reviewed to include only citations in which the concept being searched was a major topic. When the search strategy was reviewed such that "Randomised controlled trial" [publication type] was dropped for Randomised Controlled Trials as Topic the number of citations from the pubmed search was remarkably reduced to (336), with the following as the search terms: "Random Allocation"[Mesh] AND "Randomised Controlled Trials as Topic"[Mesh]. The third pubmed search that contains the following terms:

"Random Allocation"[Mesh] AND "Controlled Clinical Trials as Topic"[Mesh] yields 350 citations. This new result appears to be more concise in addition to the fact that it is more objective as the search terms reflect the content of the topic more than any of the previous searches made. Thus, these latest search terms were used for the purpose of this review. Having carefully gone through all the 350 citations by topics, 92 of them were initially identified as having possible relevance to different aspects of the topic under review. However, a close scrutiny of the abstracts revealed that only 22 articles were relevant for the purpose of this study. Full texts of the 22 articles identified were accessed and four other relevant articles got outside the search strategy described were also included.

The review result was structured under two main headings: Random allocation and how not to do it, and random allocation techniques

\section{Random allocation and how not to do it:}

The randomised controlled trial has been widely described and accepted as the best trial design in the investigation of medical therapies [3] since conscious scientific efforts are made to reduce bias; foremost among such bias reducing techniques is randomisation. Randomisation is about chance allocation of participants to treatment groups; thus, in addition to reducing bias, it also permits a valid test of significance. The successful implementation of this all-important procedure, as was observed by previous authors [1] depends on two main procedures: (1) generation of an unpredictable random allocation sequence, and (2) concealment of that sequence until assignment occurs. The benefits of randomisation can be greatly undermined if the allocation sequence is not properly concealed and implemented. For this reason, researchers have suggested, among other things, that the person who generates the sequence should not be the person who determines eligibility and the entering in of patients to the trial; they also advocate the possibility of using people not involved in the trial for treatment allocation - 'third party randomisation' [4-5]. Schulz in his article, described allocation concealment as a procedure undertaken to conceal allocation to study group from those responsible for assessing patients for entry in the trial. Such measures include; central randomisation, sequentially numbered opaque sealed envelopes, numbered or coded bottles or containers, drugs prepared by the pharmacy, or other descriptions that contain elements that provide convincing evidence of concealment. He further stressed that allocation that is not concealed would not take adequate measures to conceal group 
assignment from those that have the responsibility of enlisting participants into the trial. An unclear allocation concealment results when authors did not report or provide inadequate description of the method used, such that it becomes difficult to classify it as concealed or unconcealed. Allocation concealment is not the same as blinding; while it is possible to conceal the randomised sequence in all randomised controlled trials, blinding is only possible in some.

On the other hand, there have been some misrepresentations of the concept of randomisation, both in principle and in practice [2]. Although randomisation is supposed to be fundamental to the success of any controlled clinical trial, it remains perhaps the least understood. Many researchers confuse nonrandom techniques, such as haphazard and alternating assignment, with true randomisation techniques [1]. It has been argued [6] that whilst randomisation could not have been a better procedure in controlling selection bias than assignment based on strict alternation, the reason for the preference given to the former procedure was because of its ability to conceal the allocation sequence. Chalmers stressed that the much published streptomycin trial offers a methodological model in this respect, as allocation was based on randomisation - representing a shift from alternation. Non-reporting of the allocation technique in some controlled clinical studies could be because the methods used by the authors of such studies were short of a true random process.

In separate reviews of medical journals for the adequacy of randomisation procedures in trials, $[1,7]$ respectively found $129 / 206(63 \%)$ and $79 / 232$ (34\%) of authors did not specify the method used to generate an allocation sequence, despite a Consolidated Standard of Reporting Trials (CONSORT) [8] statement that stipulates that authors should make clear how randomisation was conducted. The result of their review also shows that non-random methods such as using case record number, date of birth and date of presentation are still being confused and presented as a random method by some researchers. However, it is worth noting that a considerable reduction in the proportion of medical researchers that defaulted on the CONSORT statement was observed in the two reviews. This implies that compliance with the regulatory statement actually grew between the times of the two reviews.

Despite all the benefits that randomisation confers on controlled clinical trials, its applicability in some studies has been challenged. In one of such studies [9], the author argued that a well-conducted observational study could be better than a randomised controlled trial in the investigation of endoscopic therapy. The basis of his argument was that a randomised controlled trial is accepted as the gold standard in the design of medical studies with randomization a fundamental activity; it becomes dangerous therefore to put together a randomised controlled trial with distorted randomisation. A distorted randomisation in a trial can actually show a treatment effect that is as a result of biased allocation; it was reckoned that such trials are more dangerous than observational studies as statistical considerations and overall interpretations usually take into account bias in non-experimental studies [10].

\section{RANDOM ALLOCATION TECHNIQUES:}

\section{Simple randomisation}

Simple randomisation is a procedure that ensures that trial participants have an equal chance of being allocated to a treatment group. It has been identified as the most basic of all sequence generation techniques. Different authors $[1,11]$ have observed that this elementary and basic allocation procedure surpasses all other sophisticated and complex allocation techniques in its unpredictability of allocation sequence and control of bias. The techniques of sequence generation by simple randomisation procedure in a controlled clinical trial setting can easily be facilitated. Such techniques include; tossing of coin, die throwing, card shuffling and using table of random numbers. While using a table of random number for equal allocation to two groups, researchers have several options by which the random numbers could be read. The starting point is actually arbitrary and the direction should be predetermined and adhered to strictly. However, a major drawback of the simple randomisation procedure is that treatment groups can, by chance, end up being dissimilar both in size and in prognostic factors. These imbalances might be substantial in small sample trials. The implication of this is that post-treatment crude comparison of effect between the groups might produce a biased estimate and hence a misleading trial result [7]. This explains the reasons for using mechanisms, both at the design and statistical analysis stages, to attain balance in treatment groups prior to final comparison of effect.

Researchers are of different opinions on the subject of handling chance imbalance by simple randomisation, especially at the design stage [1215]. In their [7] article: "Is Restricted randomisation necessary", while commenting on 
the reason for stratification on important covariates, argue that simple randomisation is safe and that there is no need for stratified randomisation since covariate imbalances can be adjusted statistically. Hewitt and Torgerson draw the strength of their claim from a previous study [16], where the author noted that stratification followed by an adjusted analysis does not add much to the statistical power to detect treatment effect. It was observed [1] that the inherent baseline imbalance with simple randomisation becomes negligible in large sample trials; here ( $\mathrm{n} \geq$ 200).

\section{Blocking}

Given the potential failure of a simple randomisation procedure to ensure balance in the number of participants in the respective treatment arms, especially in small size trials, the first design method that aims at making the groups balanced in size is blocking. Block randomisation, also called random permuted blocks, belongs to the family of restricted random allocation procedure. It is the most frequently used method for achieving balanced randomisation at the design stage [1]. Balanced randomisation strives for unbiased comparison groups, but also strives for comparison groups of about the same size throughout the trial [17]. This attribute becomes helpful when investigators plan interim analyses; it makes a meaningful treatment effect comparison possible between groups at such time when there are indication that the trial might be terminated before the final recruitment. Smaller block size(s) is/are used to ensure balance in the treatment groups.

For example, with a block size of 4 , there is an assurance that the group is balanced each time the $4^{\text {th }}$ patient is enrolled. With a block of size 4 , there are six ways in which we can allocate treatments so that two subjects get $A$ and two get $B$ [18]: $\mathrm{AABB}$ 2.ABAB 3.ABBA 4.BBAA 5.BABA 6.BAAB

However, a note of caution while using this method is that the blocking arrangement used should not be revealed to the clinic personnel until it is appropriate to do so. However, because of the possibility of sequence prediction, especially in unblinded trials and when the block size is so short (and fixed), the use of two or more block sizes for a trial has been suggested [19].

Often, stratification is done in combination with blocking $[1,7,18]$. At such times, the usefulness of blocking can be reduced by the use of too many allocation strata.

\section{Stratified random allocation}

This is a procedure that divides the trial participants into strata according to important outcome-related prognostic factors; following classification an allocation procedure takes place within each stratum by which participants are assigned to treatments groups using separate randomization schedules. During this process, it has been advised that the investigator use some form of restricted randomisation - usually blocking to generate the allocation sequence [7]. Simple randomisation often fails to produce balance in baseline prognostic factors between treatment groups, especially when the sample size is small; stratified random allocation represents the first design effort to correct for this. It has been argued [20] that the estimate of treatment effect can be more precise and has more power following a stratified random allocation procedure in a trial with small sample size; this is obvious since stratification reduces variance. Both power and precision are inversely related to the variance. These authors further identified benefits of stratified randomisation to include; increased efficiency, facilitation of subgroup analysis and protection against type I error. When considering the effect of stratification on power, they found that the adjusted analysis with pre-stratified randomisation led to increase in power compared to the unadjusted analysis in which there is no stratification. They argued that the greatest increase occurred for therapies with large treatment effect [14].

However, associated inherent complexity has limited the application of this procedure in the design of randomised controlled experiments. For example, it breaks down when there are several important prognostic factors to account for at the design stage of the experiment. In addition, in large trials, stratified randomisation confers little or no gain, especially for the complexity it usually presents with; instead some researches will prefer to use simple randomisation procedure for treatment assignment $[1,11]$ and then account for the imbalance at the analysis stage. They also argue that the gain from stratification becomes minimal when participants in each of the treatment groups are more than 50. It should also be noted that a common rule of thumb when stratified randomisation is used is to always include the stratification factor in the adjusted analysis [15]; even though we do not need to stratify to statistically account for important prognostic factors in trial [7]. It has also been argued that for a sample size above 50, stratification followed by 
adjustment does not add much statistical efficiency or power to detect a treatment effect [16].

Another issue of much importance in stratified randomisation is the choice of the appropriate number of strata; the general consensus on this is to make the number of strata few so as to keep the trial manageable $[1,18]$. However, it has been observed by various authors that in practice it is rarely possible to stratify for more than two factors, especially in small trials $[18,21]$. Previous authors [22] suggested that the number of strata be appreciably less than $n / B$, where $n$ is the total sample size and B is the block size. Regarding appropriate number of strata in stratified randomization, [20] modified earlier recommendations by Hallstroom and others: they suggest that the number of strata should not be more than $\mathrm{n} /(\mathrm{Bx} 4)$, where $\mathrm{n}$ is the sample size at the first planned interim analysis and 4 is a safety factor that accounts for unequal distribution of patients among strata. They further illustrated this by given example of a trial of 1000 patients with random allocation of block size of 4 and with the first interim analysis planned after 500 are enrolled; following their formula for calculating the optimum number of strata, then there would be a maximum of 31 strata [20].

\section{Minimization}

Minimization is the first covariate-adaptive randomisation procedure, proposed in the 1970s [23]. It is a covariate-adaptive allocation procedure because conscious effort is made to create balance in covariate distributions between groups even where stratification fails [12]. Minimization is an alternative allocation procedure to stratified randomization in the quest to balance prognostic factors between the treatment groups [20-21]. Here, a random allocation technique is used to assign treatment to the first patient [24] and subsequently the decision on which patient would result into better balance in the groups is made with respect to a particular prognostic factor. Minimization uses information about patients who are already in the trial to determine treatment assignment for the incoming participant such that differences between groups are minimized [20].

There are different types of minimization procedure; Taves's minimization method makes use of the information on the previous participant group assignment to decide on the next assignment until all the enrolled participants have been completely assigned [25]. Thus, the next patient is usually assigned to the treatment group with the lower covariate marginal total. As this feature makes the allocation of the next subject to treatment group dependent on the balance of the groups, this procedure is sometimes referred to as dynamic allocation [23]. Medical researchers have variously submitted that minimization offers a solution to the limitations of stratification at balancing for multiple prognostic factors in small trials as the procedure makes treatment groups similar in several important features even with small samples $[1,18,21,25]$. It was observed [13] from the result of a simulation study that minimization provides better balanced treatment groups when compared with unrestricted randomisation and that it can incorporate more prognostic factors than stratified randomization methods. They however also added that adjustment should always be made for minimization factors when analysing trials that used minimization as the allocation method; recognizing that minimization is not a random method but that tests of statistical inference are based on the assumption of random assignment to treatment and control groups. This agrees with the view of earlier authors on the subject [26]. The view that minimization is not entirely a probabilistic procedure was also shared by different authors $[1,12]$.

However, it has also been noted that this procedure may lead to predictability of assignment $[7,25]$; thus, it was suggested that researchers could add a random element to the procedure [7], perhaps by randomly allocating the first few participants [25]. Other drawbacks inherent within minimization include the complex computation process involved; however, a user-friendly program that manages this has been developed and is available.

\section{CONCLUSION}

Generation of random sequence and concealment are crucial to a successful random allocation. Even though generation of allocation sequence by simple randomization is easily facilitated, a major drawback of the technique is that treatment groups can, by chance, end up being dissimilar both in size and composition of prognostic factors. More complex allocation techniques yield more comparable treatment groups but also present with certain challenges. However, it is important that whichever allocation technique is employed, unpredictability of random assignment should not be compromised. 


\section{ACKNOWLEDGEMENT}

This study is based on my $\mathrm{PhD}$ work in biostatistics, and I wish to express my gratitude to the Research Institute of Primary Care and Health Sciences, Keele University, United Kingdom, for providing full funding through bursary and scholarship awards and an enabling environment for a successful completion of the PhD. I also want to express my profound gratitude to Prof. Julius Sim and Dr Martyn Lewis for supervising the work.

\section{REFERENCES}

1. Schulz K F, Grimes DA. Generation of allocation sequences in randomised trials: chance, not choice. THE LANCET 2002;359: 515-519.

2. Liu J P, Kjaergard LL and Gluud C. Misuse of Randomization: A review of Chinese randomized trials of herbal medicine for chronic hepatitis B. American Journal of Chinese Medicine 2002; 1 : 173-176

3. Mcleod RS, Wright J.G. Solomon M.J, Hu X, Walters B.C, and Lossing A.I Randomised controlled trials in surgery: Issues and problems. Surgery 1996; 119: 483-6.

4. Altman DG, Schulz KF. Concealing treatment allocation in randomized trials. BMJ 2001; 323: 446-447

5. Schulz KF. Assessing allocation concealment and blinding in randomised controlled trials: why bother? EBN Based Nurs 2001; 4: 4-6.

6. Chalmers I. Why transition from alternation to randomization in clinical trials was made. BMJ 1999; 319:1372.

7. Hewitt C E, Torgerson D. Is restricted randomization necessary? BMJ 2006; 332: 15061508.

8. Moher D, Schulz KF, Altman D. The CONSORT statement: revised recommendations for improving the quality of reports of parallel-group randomized trials. JAMA 2001; 285 :15: 1987-1991

9. Cotton PB. Randomization is not the (only) answer: a plea for structured objective evaluation of endoscopic therapy. Endoscopy 2000; 32 :5: 402-5

10. Torgerson DJ, Roberts C. Randomization methods: concealment. BMJ 1999; 313; 375-376.

11. Lachin JM, Matts JP, Wei LJ. Randomization in clinical trials: conclusion and recommendations. Control Clin Trials. 1988; 9:365-374

12. Rosenberger WF, Sverdlov O. Handling covariates in the design of clinical trials. Statistical Science 2008; 23:3: 404-419.

13. Scott NW, McPherson GC, Ramsay CR, Campbell MK. The method of minimization for allocation to clinical trials: a review. Controlled Clinical Trials. 2002; 23: 662-674.

14. Kernan WN, Makuch RM. Letter. J Clin Epidemiol 2001; 54: 104-107.
15. Hagino A, Hamada C, Yoshimura I, Ohashi Y, Sakamoto J, Nakazato J. Statistical Comparison of random allocation methods in cancer clinical trials. Controlled Clincal Trials 2004: 25; 572-584

16. Grizzle J E. A note on stratifying versus complete randomization assignment in clinical-trials. Control Clin Trials 1982; 3:365-368

17. Schulz KF. Randomised controlled trials. Clin Obstet Gynecol 1998; 41:245-256

18. Altman DG, Bland J.M. How to randomise. BMJ 1999; 319:703-704

19. Diog GS, Simpson F. Randomization and allocation concealment: a practical guide for researchers. Journal of critical care 2005; 20:187193

20. Kernan WN, Viscoli C M, Makuchi RW, Brass LM, Horwitz, RI. Stratified randomization for clinical trials. Journal of Clinical Epidemiology 1999;52: 19-26.

21. Roberts C, Torgerson D. Randomization methods in controlled trials. BMJ 1998; 317:1301.

22. Hallstroom A, Davis K. Imbalance in treatment assignments in stratified block randomization. Control Clin Trials 1988; 9: 375-382

23. Cai H, Xia J, Xu D, Gao D, Yan Y. A generic minimization random allocation and blinding system on web. J Biomed Inform 2006; 39: 6: 706719

24. Altman DG, Bland JM. Treatment allocation by minimization. BMJ 2005; 330:843

25. Minsooo K, Brian GR, Park J. Issues in Outcomes Research: An overview of randomization techniques for clinical trials. Journal of Athletic Training 2008; 43: 2:215-221.

26. Senn S. A personal view of some controversies in allocating treatment to patients in clinical trials. Stat Med 1995; 14: 2661-2674. 